\title{
Les années 2012. Manifestations littéraires du printemps étudiant dans le Québec contemporain
}

\author{
David Bélanger \\ Université McGill
}

Si la notion de « relève » s'avère moins offensive que celle d'avant-garde, elles proviennent toutes deux, néanmoins, du lexique militaire. Ce qu'on relève, précisément, c'est la garde on connait sa variation politique : on parle de changement de garde - éreintée par de longues années à «tenir le fort », rendue placide, cynique, prête au repos et poussée à ce repos par la fougue de la relève. Qu'on ait volontiers troqué une expression pour l'autre 
s'explique par le constat postmoderne de la fin des avant-gardes, homologue à « la fin des grands récits », parmi lesquels on retrouvait en belle place la téléologie du progrès, celui de nos sociétés ou encore de nos arts. Ne plus croire au dépassement conduit inévitablement à ne plus concevoir d'avant-garde. Or, si l'avant-garde disparaît et si, donc, l'art ne se pense plus en assaut mais plutôt en défense du fort dont on relève la garde, est-ce à dire que la « valeur » de l'art et de la littérature a elle aussi mué ? Comme le mentionne le sociologue Michel Freitag : «On assiste ainsi à cette fuite en avant de 1'“avant-garde”, qui est en réalité l'arrière-garde qui s'efforce de maintenir à tout prix dans la société moderne la "place", le "lieu" de l'art classique, et donc de protéger l'art contre la disparition de son concept. » (2002, P.131.) La garde qu'on doit relever de génération en génération, s'occupe à garder quoi au juste ?, voilà ce qu'on devrait demander. La réponse de Freitag et d'autres théoriciens et théoriciennes contemporains se résume à la chose elle-même ${ }^{1}$ : un concept en soi, une sorte de scène d'énonciation privilégiée, une autonomie n'ayant plus l'ambition ni de se transcender dans la société, ni de se révolutionner ou même de se renouveler.

Cet état des arts et de la littérature dans le discours social, ou à tout le moins dans le discours universitaire, a porté mes travaux sur la littérature québécoise de la dernière décennie : en analysant des œuvres parues depuis l'an 2000, j'observais que chez plusieurs auteurs, fortement inspirés par les études littéraires, la littérature devenait une classe restreinte réduisant à la fois la portée de l'énoncé littéraire à l'entendement des seuls lettrés

\footnotetext{
${ }^{1}$ De tels « oracles » s'avèrent pour le moins nombreux et divers, allant de Dominique Maingueneau et son Contre Saint-Proust, au collectif récent paru aux éditions Codicille, D'une littérature qui se fait à une littérature qui se meurt? en passant par le collectif français Fins de la littérature (2011) ou encore les travaux de William Marx, L'adieu à la littérature (2004) et La haine de la littérature (2015).
} 
DAVID BÉLANGER, «Les années 2012. Manifestations littéraires du printemps étudiant dans le Québec contemporain »

et à la fois le rôle de la littérature sur la place publique ${ }^{2}$. Ces constats répétaient le lien tranché entre la société et le discours littéraire, affirmant non sans dépit l'absence de tout engagement social, de toute volonté à s'engager d'une part, et même le voulant, de tout pouvoir dans la sphère médiatique d'autre part. C'est à la radicalité d'une telle affirmation que j'aimerais m'attaquer dans le présent article, en effectuant une sorte d'auto-critique comme le disaient les marxistes. Le printemps étudiant de 2012 constituera alors le prisme par lequel appréhender la question du possible engagement de la littérature dans le discours social.

Devant l'ampleur des textes produits durant le et autour du printemps étudiant, il me faut déjà établir une hypothèse restrictive. Le printemps 2012 a formé une clameur, un collectif taillé dans la polarité politique. L'enjeu principal aura été, du côté du pouvoir, de s'arroger le vocabulaire contre les manifestants et dissidents ; en contrepartie, on a tâché, du côté des manifestants, d'ouvrir le langage à la multiplicité des voix et des possibles. Cet enjeu constituera le fil que suivra le présent article, en ce que la question lexicale et idéologique se trouve réinvestie par la littérature. Dans un premier temps, j'expliciterai, par un rapide survol, l'organisation discursive autour du printemps 2012, après quoi il s'agira de s'attarder plus spécifiquement, dans des œuvres de fiction - celles de Stéfanie Clermont, Perrine Leblanc, Cassie Bérard et Patrick Nicol -, sur la position du militant par rapport au groupe. Par voie de conséquence, l'attention des analyses se portera sur la place du discours

${ }^{2}$ Parmi mes travaux sur le sujet, au cœur de ma thèse déposée à l'Université du Québec à Montréal (2018), je voudrais référer plus particulièrement à mon article «Trois écrivains au café. Interactions et socialités dans la littérature québécoise contemporaine », @nalyses, vol 12, no 3, automne 2017, p. 42-74 ou encore à un chapitre de livre : «La littérature comme classe sociale : figuration de la littérature dans le roman québécois contemporain », dans Robert Dion et Andrée Mercier [dir.], Que devient le roman québécois? Formes et enjeux des pratiques narratives depuis 1990, Montréal, Nota Bene, p. 339-363. 
littéraire en regard de l'idéologie militante. Après tout, le littéraire comme norme, comme code de classe, se pose difficilement sur la parole démocratique. Cette difficulté, comme piste à suivre, ne devra pas être perdue de vue.

\section{Une abondance à interroger}

Il convient d'entrée de jeu de mettre le multiple des paroles en contexte : en février 2012, après l'annonce d'une importante hausse des frais de scolarité universitaire, plusieurs associations étudiantes québécoises entrent en grève. Tenace, le mouvement étudiant gonfle plutôt que de s'affaiblir dans le temps, engageant une véritable polarisation dans l'espace public entre, d'un côté, une jeunesse « rebelle », « révolutionnaire », « enfants gâtés » et de l'autre, un gouvernement «méprisant», « inflexible», volontiers «paternaliste ». Dire qu'on a beaucoup écrit durant le printemps étudiant relève forcément de l'euphémisme : dans les journaux ${ }^{3}$, dans des blogues, sur les réseaux sociaux, sur des affiches, en revue et, dans un geste étonnant d'engendrement de l'histoire, en livres, avec des anthologies, des essais, des explications, des dictionnaires, le printemps 2012 a été nimbé de mots et de définitions.

Pour illustrer les enjeux de cette prise de parole, l'exemple de la revue Fermaille, fondée durant le printemps 2012 par, notamment, des étudiants de littérature en grève, permet d'en saisir l'esprit. Très vite mis en « anthologie » (dès 2013), les intervenants de la revue parlent constamment des conditions de la parole lettrée durant le mouvement. Dans son analyse de Fermaille, Mélissa Labonté en fait même «le véritable enjeu qui anime la revue »: «l'apparent mutisme de la littérature face à cette aliénation du sujet des enjeux

${ }^{3}$ Je renvoie ici au mémoire de Jean-Philippe Roy, qui se propose de faire une sociocritique du printemps étudiant dans le Journal de Montréal : Le printemps érable au Journal de Montréal : figures de l'imaginaire social et mise en récit, Université de Montréal, 2016. 
collectifs. » (Labonté, 2017, p. 74) Ainsi, on retrouve, au gré de l'anthologie, des textes au fort contenu métadiscursif, de Jean-Philippe Chabot qui rappelle que «nous nous sommes donnés comme mandat initial d'agir selon une logique pulsionnelle pour rendre au conflit actuel la pluralité de ses souffles » (2013, p. 135) à Catherine Lavarenne, soulignant le marasme que crée l'en-trop de textes :

J'ai un problème de lecture. [...] Il y a des arguments qui poussent sur les branches et des contre-arguments qui pleuvent jusqu'aux racines. Il y a du texte partout et j'en ai plein la vue. [...] Je n'en peux plus de m'entendre écrire. Il faut que ça se passe. (2013, p. 149)

Il existe, on le constate, une dialectique dans la revue, qu'on voit répétée tout au long de l'anthologie : celle entre le désordre d'une foule - on pourrait même dire, d'une clameur -, et l'ordre logique, mais par là mortifère. En effet, chez Chabot, l'oxymore «logique pulsionnelle » nomme cette libération de la logique, comme chez Lavarenne, la bataille rangée de la logique argumentative crée l'exaspération dans l'action, « il faut que ça se passe ». Ce nœud oxymorique n'est pas anecdotique dans le discours du printemps 2012 : Patrick Turmel et Jasmin Miville-Allard, en préface à l'anthologie Fermaille, parlent de la «langue au Québec [qui] est brouillée, récupérée, modifiée par la novlangue toujours abjecte $»$ (2013, p. 9). Lacroix, Nadon et Parenteau, dans leur analyse de la place de la littérature dans le printemps 2012, traitent d'un « détournement de langage systématique et cynique de la part du gouvernement et de ses alliés médiatiques [qui] a mis en évidence la face guerrière de la langue de bois et sa violence symbolique ». (2014, p. 233) C'est une langue rigide, réifiée, contre laquelle doivent batailler les grévistes - il parait alors naturel qu'on oppose à cette rationalisation des échanges une «action» ou une «logique pulsionnelle ». 
«Pour la Raison, ce qui n'est pas divisible par un nombre, et finalement par un, n'est qu'illusion ; le positivisme moderne rejette tout cela dans la littérature » (1974 [1944], p. 25), écrivent Theodor Adorno et Max Horkheimer dans leur Dialectique de la raison. Il y a de cela derrière les positions durant le printemps 2012 ; entre « la technique comme idéologie » pour paraphraser un titre de Jürgen Habermas et la pensée des arts comme émancipation ; entre les sciences humaines, massivement en grève, et les sciences naturelles et les savoirs techniques, parfois récalcitrants ; entre, aussi bien, des espaces de parole institués et des places publiques à conquérir, voilà le nœud oxymorique configurant les paroles du printemps. Pour terminer ce contexte discursif, deux manifestations précises de l'engagement de la littérature doivent être présentées.

La première se retrouve dans le Dictionnaire de la révolte étudiante. Invités à définir un mot ou une expression importante du printemps 2012, des personnalités mais aussi plusieurs écrivaines et écrivains interprètent le mot bien davantage qu'ils ne le définissent ; le positivisme de la langue, sa rigidité (normative, mais aussi bien médiatique) s'avèrent malmenés. De fait, dès lors que la science a été mis au service des moyens de production, elle devient technique, soumission de toute activité de pensée à des finalités claires, puis enfin, unique mode de légitimation de nos usages. La langue réifiée est efficace, sans ambiguïté, positiviste et domine ce qu'on en fait, il faut la casser, la braconner pour qu'elle s'adapte au mouvement des masses. C'est donc contre cette rationalité que les écrivains s'élèvent dans leurs interprétations. Ainsi, Jean-Simon DesRochers définit le «Sondage» dans sa scientificité et son biais, dans sa technique (fortement idéologisée) et sa limite : 
Règle générale, les sondages se font à partir d'échantillons de mille personnes dotées de téléphones à ligne terrestre, de soirées libres et de temps à perdre (c'est-à-dire que les sondages sont méthodologiquement conçus pour représenter le plus fidèlement possible les idées d'une population incapable d'en produire). À cet échantillon représentatif de la vacuité intellectuelle [...] est posée une question dont la formulation conditionne l'essentiel de la réponse. (2012, p. 197-198)

À la manière de cette «logique pulsionnelle », la méthodologie comme déficience pointe une science incapable d'atteindre la pensée et les idées ; plutôt agit une véritable entreprise d'autoengendrement de l'opinable. Samuel Archibald, dans l'entrée de «la classe moyenne » parlera plus sombrement d'un « agenda néolibéral dont la stratégie première est la même que dans tous les pays industrialisés : faire de la classe moyenne l'instrument de sa propre destruction ».(2013, p. 39) Agenda, stratégie, instrument: la même rationalité étatique, la même technique comme idéologie sourd ici, mais de l'autoengendrement de l'opinion on passe à l'autodestruction de la classe. Cet exemple me permet de relever les conditions de l'engagement de la relève littéraire. En effet, devant cette entreprise conceptualisable de réification étatique du langage, le champ de bataille paraît clair, et Fermaille comme le Dictionnaire de la révolte ont agi sur ce front de façon ostensible. Un peu plus bas, je tenterai de démontrer que cette tension apparaît, sous une autre forme, dans les œuvres de fiction.

Deuxième manifestation : corrélative à la précédente, elle constitue une résistance non pas au langage, mais aux institutions. Mélissa Labonté décrit Fermaille, en ce sens, comme «publication sauvage en marge de l'institution». (Labonté, 2017, p. 92) Cette parole se retrouve à la fois «à côté » de l'Université, puisque c'est à la faveur d'une grève universitaire qu'elle se déploie, et à la fois, par son mode d'édition artisanal, à côté de l'institution littéraire. En exergue de l'anthologie, on lit ainsi, de Catherine Lavarenne : 
« Ce livre n'est pas un criss de porte-folio/c'est de la vociférance de grève. » De même, en page de garde pourra se lire : «Ce projet est autonome, autofinancé et ne bénéficie d'aucune subvention. » La parole gratuite de la littérature se radicalise dans sa formulation politique : car si toute une institution artistique a intégré les critères antiéconomiques, cela se fit par le biais d'appareils étatiques, subventionnaires, compétitifs, rendant tangibles le fameux marché symbolique théorisé par Bourdieu. Or, la parole de la foule du printemps 2012 tente précisément d'échapper au « symbolique » miné par les institutions, tout comme elle dénonce l'assujettissement économique qui menace les universités : le grand ordre est malade, il faut tout renverser. «Au regard de l'ordre, le printemps étudiant n'était qu'un carnaval », (2013, p. VII) écrit George Leroux à propos des événements. Le constat peut se penser sans sa restriction, à savoir que le printemps 2012 fut un carnaval, chargé de la force lucide du rire politique, du rire populaire détaché de son institution pour révéler l'édifice instable des pouvoirs. Mikhail Bakhtine écrit bien, dans son Euvre de François Rabelais :

Toute la très riche culture populaire du rire au Moyen Âge a vécu et évolué en dehors de la sphère officielle de l'idéologie et de la littérature élevée. Et c'est grâce à cette existence extra-officielle que la culture du rire s'est distinguée par son radicalisme et sa liberté exceptionnels, par son impitoyable lucidité. (Bakhtine, 1970, p. 80)

Il ne s'agit évidemment pas de remarquer une carnavalisation du Moyen Âge dans un mouvement politique moderne. Plutôt, il faut saisir qu'en dynamique, le printemps 2012 a tenté, comme l'énonçaient les théoriciens de l'École de Francfort, comme l'énonçait Bakhtine, comme l'énonçait toute une vulgate marxiste dénonçant l'unité (institutionnelle, 
DAVID BÉLANGER, «Les années 2012. Manifestations littéraires du printemps étudiant dans le Québec contemporain »

bourgeoise, idéologique) pour appeler la totalité (prolétaire, populaire, extra-officielle) ${ }^{4}$, de congédier un ordre au nom du désordre, renversant le standard au profit de l'humaine existence, l'idéologie normée au nom de pulsions, de souffles, de vociférances. On ne peut appréhender le rapport entre littérature - en tant qu'espace fortement institué, ordonné - et printemps 2012 en faisant l'économie de cette distinction. Et lorsque la relève appréhende ces événements sous les formes normalisées de la littérature, on ne peut que noter cette tension oxymorique entre la prise de parole de la littérature et le refus de la norme au cœur de la révolte.

\section{L'un et le groupe : le texte et la foule}

Dans leur analyse du rapport entre littérature et printemps 2012, Lacroix, Nadon et Parenteau affirment «brutalement » que les générations d'écrivains ne furent pas égales devant la grève, les autrices et auteurs ayant commencé à écrire entre 1960 et 1980 montrant de grandes réticences : «La lutte, la prise de parole collective, pour eux, c'est du passé, un passé tantôt renié, tantôt mythifié, mais surtout dépassé. Ne sont-ils pas nombreux ceux qui, dans ces générations, ont depuis opté pour la littérature "pure", qui dépend financièrement de l'État ${ }^{5}$ ? » De là, on peut dire que le printemps 2012 a cristallisé dans le discours sur la littérature une distinction générationnelle, elle-même performant l'effet relève. Or, on le sait à la lumière du dossier dans lequel s'inscrit mon propos, la relève littéraire actuelle précède de beaucoup, dans l'usage, l'année 2012, et trouve ses débuts dans le fort effet «changement de garde » engagé par les nouvelles maisons d'édition,

\footnotetext{
${ }^{4}$ Je reprends cette opposition entre la critique conventionnelle et la pensée marxiste à André Belleau, notamment dans «Littérature et politique», Y a-t-il un intellectuel dans la salle ?, Montréal, Primeur (L'échiquier), 1984, p. 97-99.

${ }^{5}$ Michel Lacroix, Rachel Nadon et Olivier Parenteau, art. cit., p. 237.
} 
l'apparition de nouvelles écuries d'autrices et d'auteurs, des manières d'écrire déplacées, réorientées, etc. Mon postulat est le suivant : le printemps 2012 fut, pour cette génération, un baptême du feu vers l'écriture engagée, un lieu où la parole fut contrainte par la rue de se repenser dans le social, de se positionner en regard de lui. Pour les besoins de la démonstration, je vais restreindre mon analyse au rapport de l'individu à la manifestation, c'est-à-dire au rapport de l'un - l'auteur, le scripteur, la subjectivité «problématique » - à l'ensemble, comme désordre.

Des œuvres au statut très différent viennent à l'esprit pour saisir le déplacement, «l'effet relève » qu'engagea le printemps 2012. Deux générations se mobilisent : d'une part des auteurs comme Patrick Nicol, auteur ayant commencé sa carrière dans les années 1990, mais aussi Catherine Mavrikakis, dont l'écriture romanesque s'ancre dans la décennie 2000 ; de même pour Élise Turcotte, qui commença à publier dans les années 1980. D’autre part, Perrine Leblanc, Cassie Bérard, David Turgeon, Stéfanie Clermont et Simon Brousseau participent de cette génération dont l'écriture prend racine après $2010^{6}$. Tout de même, le printemps 2012 est sémiotisé chez tous ces auteurs, et plus encore, leur manière de raconter sur le printemps passe par une tension discursive entre une vociférance libre mais inquiétante et une parole possédée comme savoir, dont l'usage est garant du récit.

${ }^{6}$ Je ne pourrai traiter toutes ces œuvres, mais il faut voir qu'elles sont considérées dans le constat que je tente ici de tracer. Le chantier que j'aimerais entreprendre avec cette réflexion pourrait également analyser l'essai du leader étudiant Gabriel Nadeau-Dubois, Tenir tête, et sa contrepartie romanesque, Tenir parole de Clément Courteau et Louis-Thomas Leguerrier ; la poésie de Samuel Mercier dans Les années de guerre, le rapprochement étonnant que Marie-Claire Blais opéra, dans le discours social, entre les manifestations américaines des années 1960 et la révolte étudiante de 2012 dans Passages américains ; les premiers titres de la collection Documents d'Atelier 10, notamment l'essai La juste part de Patrick Turmel et David Robichaud, Année rouge de Nicolas Langelier et Le sel de la terre de Samuel Archibald. La piste est ouverte. 
Ainsi, dans la nouvelle «Les astres » de Stéfanie Clermont ${ }^{7}$, le printemps 2012 ne constitue que la trame de fond d'un triangle amoureux : Zoé est en couple avec Laurent, mais la colocataire de la première, Céline, le méprise. L'enjeu de l'intrigue se résume à ce que Zoé et Céline sauront préserver de leur relation passée. Si Zoé appartient «au vrai monde », se levant tous les jours pour aller travailler, Céline et Laurent sont étudiants en sociologie et écrivent vaguement leur mémoire de maîtrise durant la déferlante de 2012. Le printemps se présente comme véritable carrefour entre les personnages, et ce dès son premier signe : c'est avec un « aimant carré rouge » que Céline accroche au frigo l'avis de renouvellement du bail de location. Cet avis, c'est le contrat même de l'amitié entre Zoé et Céline. Or, la nouvelle adopte le point de vue de Zoé, individu incarné. Ses compagnons paraissent, par contraste, théoriques, toujours dans de vaines luttes hypothétiques. La lutte étudiante s'en trouve singulièrement problématisée. Ainsi, on apprend que Laurent « se sent impuissant et coupable de ne pas être plus engagé politiquement, et que sa consommation compulsive d'actualités l'aide à calmer sa conscience » (LA : 60), on apprend que Céline est anarchiste, «contre les dogmes », mais qu'elle ne «meugle » ses principes que quand « cela fait son affaire » (LA : 61) ; au printemps 2012, tous deux « sont survoltés, analysent sans fin chaque déclaration de Charest ou de la ministre Beauchamp, chaque tentative d'André Pratte de justifier la violence policière [...], ils débattent du sexisme réel ou inventé de la CLASSE et de la FEUQ»(LA : 61) ; Laurent «ne s'en vante pas, mais il préfère [au piquetage des classes] continuer à rédiger son mémoire pour déposer le plus tôt possible quand tout ça sera terminé » (LA : 62). Céline appelle à «lâcher le discours de

${ }^{7}$ Stéfanie Clermont, «Les Astres » dans Le jeu de la musique, Montréal, Quartanier (Polygraphe), 2017. Désormais, les références à cette édition seront inscrites dans le corps du texte (LA : suivi du numéro de folio). 
membres légitimes de la société » (LA : 62) pour mener le Québec dans l'anarchie, etc. Le problème de cette représentation en contre-jour de la grève et de ses manifestations, c'est évidemment son caractère bavard, par là stérile, véritable métonymie de la relation conflictuelle entre Laurent et Céline. La nouvelle s'ouvre sur une réflexion philosophique de Laurent, qui confie calmer ses angoisses en relativisant sa place dans le monde, véritable grain de sable dans l'univers; elle embraye sur une querelle concernant les menstruations ; Céline enseigne à Laurent ses vertus, « le cycle menstruel rapproche de la nature » «ça a quelque chose de sacré », «tu prends vraiment conscience du fonctionnement de ton corps » (LA : 65). Cette querelle, si elle semble fort éloignée de l'engagement du printemps, en redouble la sémiotisation : c'est dans la dialectique entre le corps et les principes que se joue la lutte, et si Laurent reproche à Céline le manque de consistance de son féminisme - «Je me demande ce que tes amis queer diraient s'ils t'entendaient en ce moment, Céline. Utérus sacré, cycle de la lune, c'est quoi ces conneries essentialistes là? » (LA : 66) -, c'est encore une fois pour pointer l'inadéquation entre les principes et la chair. Seule Zoé, la focalisation de la nouvelle, paraît vraie : elle travaille dans des jardins, les mains dans la terre, ne prétend à aucune connaissance politique ou théorique. Céline et Laurent ne s'accomplissent, au jour le jour, qu'en réussissant à démonter des arguments bidons, mais en se plaçant sans cesse «au-dessus » de la mêlée, penseurs rationnels à condition d'abandonner corps et actions - de rester loin des manifestations. Cet «intellectuel bavard», parfois doctorant, fait retour dans les nouvelles de Stéfanie Clermont, manière d'appuyer la lutte en pointant l'inertie intellectuelle qu'elle recouvre. 
Dans Malabourg de Perrine Leblanc ${ }^{8}$, même phénomène : l'action du roman se situe, pour l'essentiel, en Gaspésie, à Malabourg, où la protagoniste, Mina, est témoin du meurtre d'une camarade de son âge. Il se trouve toutefois que l'assassin est le père de la victime et qui plus est, maire du village. En tant qu'autochtone, Mina est, de son côté, isolée de la société des blancs et résiste longtemps à son éthique qui l'invite à dénoncer le criminel. Elle finit par le dénoncer, toutefois; alors, le maire se suicide. Mina a déboulonné deux autorités, père et maire, pour ensuite fuir à Montréal. Son statut, là-bas, reste ambigu, elle «réécrit les dissertations d'étudiants faibles qui la rémunèrent correctement. Quand elle est vraiment à court d'argent, elle révise des mémoires et des thèses, largement subventionnées par l'État, qui seront publiées aux Presses universitaires après avoir reçu du jury d'évaluation la mention très bien. » (M : 146-147) Elle regrette parfois son travail de commis de pharmacie à Malabourg, où elle vendait quelques livres, emploi préférable à « la fréquentation dans le texte brut de ce qui deviendra l'élite universitaire » (M : 147). Au moment de la grève « la levée de cours l'arrange » $(\mathrm{M}: 160)$, elle peut se consacrer à son amant et travailler avec lui dans sa boutique de fleurs et de parfums, manier avec lui les essences, les herbes, revenir à son origine autochtone et aux traditions de sa grand-mère. Si je mentionne que son statut est ambigu, c'est bien parce que, comme Zoé chez Clermont, elle possède à la fois une autorité morale et à la fois un mépris pour les autorités officielles : Mina fait un avec les savoirs de la terre, mais agit de même comme norme linguistique aux savoirs théoriques qu'elle méprise - ces savoirs sont surdéterminés par leur institutionnalisation, largement subventionnés, évalués par un jury, élite universitaire. La

${ }^{8}$ Perrine Leblanc, Malabourg, Paris, Gallimard (Folio), 2014. Désormais, les références à cette édition seront inscrites dans le corps du texte ( $\mathrm{M}$ : suivi du numéro de folio). 
dialectique en place opère particulièrement chez Leblanc, en ce qu'il faut que le mouvement enfle et s'étende à l'ensemble de la société pour que Mina accepte d'y participer, le temps d'une manifestation - il faut, en ce sens, que le mouvement quitte les ornières universitaires, normées, institutionnelles, les questions de frais de scolarité, pour l'atteindre. Pourtant, force est de constater que Mina se définit sans cesse par ses moyens de production, de la pharmacie, à la révision à la boutique. Or, elle ne peut envisager la lutte et la protestation à l'autorité qu'une fois détachée du « fétichisme du matériel » auquel appartenait la question des frais de scolarité. Homologue, se devine la première décision consistante de la protagoniste : le maire la savait témoin du meurtre qu'il avait commis et lui avait promis de payer ses études en échange de son silence. C'est contre la gratuité scolaire, pourrait-on dire, qu'elle a fait tomber l'autorité : la gratuité des principes, leur totalité sociale, véritable nature précapitaliste dirait-on, seule compte dans la lutte.

Ces deux exemples me permettent d'expliciter un trait représentationnel des années 2012: son aspect à la fois métaphorique et allusif. Le printemps signifie le triangle amoureux chez Clermont comme il cristallise l'identité de Mina en regard des autorités. On a écrit sur Terre des cons de Patrick Nicol qu'il faisait « de la crise sociale un révélateur de crises individuelles » (Lacroix, Nadon, Parenteau, 2014, p. 238) : de même pour le roman de Cassie Bérard, Qu'il est bon de se noyer ${ }^{9}$ (2016), où le printemps 2012 est un souvenir renversé dans l'automne 2012, lors d'une grève inventée, à Asbestos, afin d'obliger la réouverture de la mine d'amiante. Il faut voir que cet usage « allusif » du printemps 2012

${ }^{9}$ Désormais, les références à cette édition seront inscrites dans le corps du texte (QN : suivi du numéro de folio). 
permet de saisir une crise au-delà de la crise. Apparait, pour parler encore une fois en marxiste, un problème général d'infrastructure. En exergue du roman de Bérard, on retrouve cette phrase tirée de Tenir tête du porte-parole étudiant Gabriel Nadeau-Dubois : «Apparemment, nous n'avons plus de rêves de liberté et de justice, nous avons des états de compte et des factures. » Se joue ici la noyade des principes dans la réalité de l'économie : payer, survivre, réussir. Cette noyade constitue par ailleurs la métaphore organisationnelle de tout le roman, alors que des enfants sont retrouvés noyés dans diverses circonstances, parfois loufoques, rappelant les coups d'une fatalité étrange mais perverse. En parallèle de ces drames que la population se garde de considérer, se développe la manifestation des travailleurs, qui constitue le nœud de la contradiction du roman : la faillite des idéaux face à l'exploitation économique permet un singulier glissement, la naissance d'un idéal de l'exploitation économique par ses sujets exploités. Se confiant à la protagoniste du roman, un leader des aspirants-travailleurs parle de ses parents et de leur implication dans les grèves de l'amiante en 1949, une grève contre l'exploitation éhontée des travailleurs, une grève réglée dans le sang, les coups de matraque d'une police étatique peu scrupuleuse : «Impossible qu'ils aient livré bataille pour rien. Il faut qu'ils aillent de l'avant avec la relance de la mine, c'est clair? ? (QN : 143) Puis d'ajouter, explicitant le lien métaphorique entre les noyades et la reprise économique: «Les investisseurs ont eu l'accord du gouvernement, mais ils se la sont tenue fermée depuis les élections. Alors, il se passe quoi ? Si le projet tombe à l'eau, on est fichus. » (QN : 143) La noyade sociale que le personnage prévoit survient plus loin dans le roman. Lors d'une manifestation, en effet, un glissement de terrain créé par un activiste envoie les manifestants au fond du trou de la mine, dans l'eau placide qui y dort, achevant de noyer les survivants. Par ce déplacement, Qu'il est bon 
www.revue-analyses.org, vol. 14, nº1, printemps-été 2019, p. 148-170.

de se noyer raconte la réification des sujets, le remplacement des idéaux par des états de compte ; lors de l'une des dernières manifestations, l'organisateur hurle dans un mégaphone : «Nous avons des choses à dire, et nous allons nous rassembler ainsi jusqu'à ce que notre voix porte. » (QN : 193) Un autre manifestant rétorquera, soulignant l'idéal manquant de cette lutte, véritable asservissement à l'industrie : «Toi qui crois que notre voix doit porter, raconte-nous. Quelles sont ces choses que nous avons à dire ? » (QN : 194)

Alain Farah mentionnait, dans l'entrée «Grève» du Dictionnaire de la révolte qu'on avait voulu « taire » le mot, «l'oblitérant avec boycott». Il rappelle toutefois ce que ce geste a d'« inculte », référant aux grèves multiples de l'histoire, celle des « ouvriers du pharaon Ramsès, canuts lyonnais, mineurs d'Asbestos. » (Farah, 2012, p. 89) Il fallait, toutes ces fois, faire front contre un ordre, contre un gouvernement, contre des états de compte trop lourds ; à Asbestos en 2012, chez Bérard, on fait front avec l'ordre et contre les idéaux, pointant de façon sibylline le fossé s'étant creusé dans les discours durant les événements. D'un côté, une clameur a, sous prétexte d'une hausse des frais de scolarité, appelé à une révolution universitaire, manière d'arracher les institutions de savoir aux exigences économiques, de ramener l'être humain - les sciences humaines - au cœur de l'enseignement et de la connaissance. De l'autre, un gouvernement et sa majorité silencieuse ont sans cesse ramené le débat sur du mesurable, rappelé l'enjeu des frais de scolarité, saucissonné la somme à défrayer par les étudiants - un café par jour, disaient-ils , et par voie de conséquence, relativisé l'importance des enseignements perçus s'ils sont détachés d'usages techniques, aisément appréhendables. Très vite, entre les idéaux et les états de compte, la discussion ne pouvait plus avancer : les œuvres répètent cette situation mais ne font-elles que cela? 
DAVID BÉLANGER, «Les années 2012. Manifestations littéraires du printemps étudiant dans le Québec contemporain »

Dans Terre des cons de Patrick Nicol ${ }^{10}$ (2012), le narrateur est fatigué, pèsent sur ses épaules les années, le sentiment de décadence, et le printemps 2012 en est l'indécidable révélateur. Lors d'une manifestation, il ne sait s'enthousiasmer comme sa blonde le fait : «C'est le carnaval, disait-elle, tu devrais être content. L'allusion à Bakhtine aurait dû me consoler. Je pensais plutôt à la maison des fous, au retour du refoulé, à la fin des haricots. » (TC : 80) Comme on l'a vu chez Clermont et Leblanc, le carnaval des manifestations constitue l'antithétique désir des protagonistes : ce sont des personnages ancrés dans la terre, dans le tangible, qui en ont tout autant contre les autorités gouvernementales que contre les théories autoritaires, toutes deux réifiées, rationnalisées, coupées d'un réel total, coincées dans l'unité des luttes idéologiques. Chez Bérard, l'inversion carnavalesque est structurelle, en ce que le geste même du carnaval se fait dans les termes de l'autorité. Si la mine n'ouvre pas, c'est moins pour des raisons économiques, infrastructurelles, que pour des raisons idéologiques, bien-pensance de gauche qui impute à l'amiante des morts au tiers-monde. La révolte s'ancre encore une fois contre un ordre rationnalisant et au nom d'une totalité existentielle, pointant bien l'aporie de toute contestation opposant principes et économie.

Juste au moment où je commence à distinguer Schubert de Schumann, Betty Goodwin de Benny Goodman, voilà que ce savoir est déclaré sans valeur. Nous avons lu Ulysse et l'Odyssée, Brief Interviews with Hideous Men et Mémoires d'une jeune fille rangée, et plus personne autour de nous ne peut soutenir une discussion moindrement prolongée. Il faudrait se maquiller, maintenant, faire des rondes... (TC: 81$)$

${ }^{10}$ Désormais, les références à cette édition seront inscrites dans le corps du texte (TC : suivi du numéro de folio). 
Voilà où la littérature coince dans les années 2012 : le narrateur de Patrick Nicol fait du carnaval des manifestations l'embrayeur d'un désordre culturel, d'une perte de valeur des savoirs à portée humaniste, comme la musique classique et la littérature. L'engagement des écrivains dans le printemps 2012 se traduit par cette sémiotisation ambigüe du carnaval, comme rejet de la culture sérieuse, rejet de la Raison comme fin première, rejet du grand ordre économique. Le travail formel, l'investissement du code littéraire dans le matériau des textes ne fait pas de doute dans ces œuvres, mais le malaise de cet investissement apparaît parfois, engageant une contradiction dans la fibre même des intrigues. Il existe une antinomie structurelle en littérature, que les œuvres des années 2012 permettent de voir réactualisée.

\section{La dialectique : symptôme et remède}

Un écrivain québécois, d'illustre génération, mentionnait lors d'une causerie à la librairie Le Port-de-Tête qu'un événement comme le printemps 2012 ne faisait pas une génération, c'est-à-dire qu'à la différence de la Guerre du Vietnam ou de la Seconde Guerre mondiale, un carnaval dans les rues ne créait pas la cohésion nécessaire pour parler de génération. Or, un peu à la manière de Mai $68^{11}$, le printemps 2012 a engagé une confrontation sur la notion même d'autorité ; par le carnaval, il a permis précisément de pointer les normes sociales, de les déplacer, de les interroger. Le leitmotiv qui a été déplié est celui d'un retrait

${ }^{11}$ Loin de moi, cependant, l'idée de rapprocher les deux phénomènes qui n'eurent pas une portée identique dans le discours social. Il est toutefois intéressant de suivre l'itinéraire intellectuel autour de Mai 68, avec sa portée dans le développement des études structurales, mais aussi d'une philosophie abstraite voyant, à l'instar de Louis Althusser, « la lutte des classes dans la théorie » plutôt que dans de véritables rapports de force: "Tout cela pouvait donner lieu à de beaux colloques ", écrit Jacques Rancière, avec un apparent dépit. Comme les années 2012, Mai 68 permet un arrêt sur l'état du savoir dans l'économie universitaire et par extension, dans l'ensemble de l'économie du savoir. Dans La leçon d'Althusser, Paris, Gallimard (Idées), 1974, p. 135. 
généralisé d'une raison - comme abstraction, théorie -, au profit d'une incarnation - dans la terre, les désirs, la rue. Cela a pris deux formes qu'il convient d'expliciter.

D'abord, les autrices et auteurs de la relève ont souligné que la révolte échappe ou doit échapper au savoir normé. Chez Nicol, le narrateur ne cache pas son mépris affectueux pour Alex, l'étudiant passionné qui se jette dans la grève, avec son vocabulaire bringuebalant et sa naïveté idéaliste. À la télévision, tenu de répondre d'un acte de violence devant le Chroniqueur, Alex «pensait que parler franc et regarder la caméra dans les yeux suffirait... Toute cette merde à propos de l'authenticité, de la sincérité, on la lui avait inculquée depuis le berceau, et il y croyait, sans mesurer l'importance du calcul et de la compétence » (TC : 76). Le point de vue du roman de Nicol est celui d'un esthète, lettré, formé au cynisme et à la raison : le roman raconte le délitement du savoir normé qui est le sien, au profit d'une authenticité bègue, écrasée par la machine rationnelle. De même, chez Bérard, la manifestation est pure parole, pure présence, coupée de tout autre principe que le besoin immédiat ; chez Leblanc, la narratrice exprime ce besoin quasiment de révolte, alors qu'elle manifeste, une unique fois, chargée de peinture rouge, en vue d'un vandalisme. Il faut ajouter que dans Malabourg, la révolte de Mina est anormale, en ce que ce personnage ne cesse de corriger la langue, des thésards aux bacheliers, en passant par les manifestants et leurs tags à l'orthographe inventif. Chez Clermont, enfin, la représentation apathique de la révolte s'ancre dans un travail de raison normée, dévalorisée comme raison en contraste à l'incarnation de Zoé, focalisatrice du récit. Ce travail, dans les textes, permet de saisir un paradoxe à l'œuvre. La littérature est un savoir normé qui peut, au mieux, médiatiser la révolte des masses, mais ne sait en reproduire la vociférance. Voilà pourquoi la norme littéraire et linguistique apparaît sans cesse, à la fois comme mode de valorisation de 
l'énonciation et à la fois comme support contrastant - scène énonciative - entre l'un et la foule. À cet égard, les œuvres se proposent en tant que «symptômes » du printemps 2012; elles se voient contraintes, dans la polarisation en place, d'intégrer la pulsion dans la logique, la clameur dans la grammaire, les gestes dans leur idéologie, les expériences culturelles dans leur hiérarchie. La littérature ne sait que réifier dans la norme littéraire l'ensemble des expériences.

Néanmoins, la même représentation prend la forme d'un «remède» dans les œuvres des années 2012 - il y va de l'esthétique critique que théorisait Adorno. L'art, en effet, est à la fois «substance objective » rendant compte d'un état idéologique et à la fois «force de protestation de l'humain contre la pression des institutions» (Assoun, 2012, p. 126). Cette protestation, dans les œuvres analysées, se dévoile comme résistance aux normes elles-mêmes exprimées. Le geste du roman de Cassie Bérard consiste à unir, dans leur antithèse, le mouvement étudiant et le développement économique d'une région québécoise ; pour ce faire, le récit doit détourner les causalités, replacer les aspirations des masses, et convenir à de nouveaux frais que c'est le détachement - l'abstraction - de l'économie qui aliène les sujets. La nouvelle de Stéfanie Clermont souligne la fragilité des principes et des logiques, pour pointer l'absolue vérité des modes de vie, des boulots routiniers, des manières de passer le temps ; c'est un sillon que le recueil approfondit de texte en texte, un peu, dirait-on, contre le savoir universitaire. La littérature se présente alors, malgré sa substance objective, comme déplacement des points de vue, recadrage des enjeux, tentative de réincarnation d'un réel réifié. 
DAVID BÉLANGER, «Les années 2012. Manifestations littéraires du printemps étudiant dans le Québec contemporain »

Forcément, le parcours ici esquissé est inachevé. Il permet cependant de faire état d'une forme d'engagement à distance, d'une littérature des années 2012. Lacroix, Nadon et Parenteau soulignaient l'attachement d'une génération d'autrices et d'auteurs, ceux ayant commencé à écrire dans les années 1960-1980, à un art d'institution, protégé des tumultes. La relève des années 2012 vient aussi d'une institution, ou du moins est traversée par elle, il s'agit de l'institution universitaire. Deux topoï collent à cette dernière : elle est détachée du social - on parle de tour d'ivoire -, et elle s'engonce dans un jargon. Le printemps 2012, avec ses « universités populaires », ses « lettres ouvertes », ses manifestations artistiques, a obligé à descendre de la tour pour parler à tout le monde. Que ce geste fasse génération, marque une relève, voilà qui importe peu à l'analyse : il se trouve que de façon politique, la question de la place du savoir a été posée dans le milieu universitaire jusqu'au milieu littéraire, et qu'elle se réfracte, de façon elle aussi politique, dans les œuvres des années 2012 pour former une sorte de résistance à. Il se peut alors que la littérature trouve dans cette brèche un nouveau lieu pour se définir et se justifier dans l'imaginaire social.

\section{Bibliographie}

ADORNo, Theodor W. et Max HorkHEIMER (1974 [1944]), La dialectique de la Raison, traduit de l'allemand par Éliane Kaufholz, Paris, Gallimard (Tel).

ARCHIBALD, Samuel (2013), «Classe moyenne », dans Le sel de la terre, Atelier 10, Montréal.

Assoun, Paul-Laurent (2012), L'école de Francfort, Paris, PUF (Quaridge).

BAKHTINE, Mikhaïl (1970), L'œuvre de François Rabelais et la culture populaire au Moyen Âge et sous la Renaissance, traduit du russe par Andrée Robel, Paris, Gallimard (Tel).

BÉRARD, Cassie (2016), Qu'il est bon de se noyer, Montréal, Druide (Écarts). 
BONENFANT, Maude, Anthony GlinOER et Martine-Emmanuelle LAPOINTE [dir.] (2013), Le printemps québécois. Une anthologie, Montréal, Écosociété.

Clermont, Stéfanie (2017) Le jeu de la musique, Montréal, Quartanier (Polygraphe).

ColLeCTIF (2012), Dictionnaire de la révolte étudiante, Montréal, Tête première.

Collectif (2013), Fermaille. Anthologie, Québec, Moult édition.

DESROCHERS, Jean-Simon (2012), «Sondage », dans Mariève ISABEL et Laurence-Aurélie THÉROUX-MARCOTTE [dir.] Dictionnaire de la révolte étudiante. Du carré rouge au printemps étudiant, Montréal, Tête Première, p.197-198.

FreItAG, Michel (2002), L'oubli de la société: pour une théorie critique de la postmodernité, Rennes, Presses universitaires de Rennes.

HABERMAS, Jürgen (1973 [1968]), La technique et la science comme «idéologie », traduit de l'allemand par Jean-René Ladmiral, Paris, Gallimard (Tel).

ISABEL, Mariève et Laurence-Aurélie ThÉROuX-MARCOTTE [dir.] (2012), Dictionnaire de la révolte étudiante. Du carré rouge au printemps étudiant, Montréal, Tête Première.

Labonté, Mélissa (2017), Faire maille. L'engagement poétique de la revue Fermaille au printemps 2012, Longueuil, L'instant même.

LACROIX, Michel, Rachel NADON et Olivier PARENTEAU (2014), « La grève en vers et en prose : combats, silences et fissures », dans Marcos Ancelovici et Francis Dupuis-Déri, Un printemps rouge et noir. Regards croisés sur la grève étudiante de 2012, Montréal, Écosociété, p. 233-255.

LEBlanc, Perrine (2014), Malabourg, Paris, Gallimard (Folio).

LEROUX, George (2013), «Préface. De la révolte à l'archive », dans Maude Bonenfant, Anthony Glinoer et Martine-Emmanuelle Lapointe [dir.] Le printemps québécois. Une anthologie, Montréal, Écosociété, p. VII.

RANCIÈRE, Jacques (1974), La leçon d'Althusser, Paris, Gallimard (Idées).

NICOL, Patrick (2012), Terre des cons, Montréal, La mèche.

RoY, Jean-Philippe (2016), qui se propose de faire une sociocritique du printemps étudiant dans le Journal de Montréal : Le printemps érable au Journal de Montréal : figures de l'imaginaire social et mise en récit, Université de Montréal. 


\begin{abstract}
Résumé
Cet article interroge la notion d'engagement au cœur du mot " relève » en art et en littérature. Que relève-t-on, au nom de quelles valeurs et de quels impératifs ? L'hypothèse suivie est à l'effet qu'après un changement de garde en littérature québécoise (autour du milieu de la décennie 2000), la littérature se relève véritablement avec le printemps 2012 un ordre de valeur et de représentation se modifie. Afin de suivre cette piste, l'article s'attarde dans un premier temps à la formation d'un discours social démocratique et antiinstitutionnel tel que rendu lisible dans Fermaille ou encore le Dictionnaire de la révolte étudiante. Dans un deuxième temps, il s'agit d'observer dans des œuvres représentant le printemps étudiant comment l'idéologie anti-institutionnelle peut s'accompagner d'un investissement littéraire. Dans Le jeu de la musique (Stéfanie Clermont), Malabourg (Perrine Leblanc), Terre des cons (Patrick Nicol) et Qu'il est bon de se noyer (Cassie Bérard), on observe que cette relève idéologiquement marquée est contredite par la formation littéraire dont elle est issue.
\end{abstract}

\begin{abstract}
Abstact
This article questions the notion of commitment to the word « relève » in art and literature. What do we see in the name of which values and which imperatives? The hypothesis followed is that after a change of guard in Quebec literature (around the middle of the 2000s), the literature really " se relève " with the spring of 2012 - an order of value and representation is changing. In order to follow this path, the article focuses initially on the formation of a democratic and anti-institutional social discourse as made readable in Fermaille or the Dictionnaire de la révolte étudiante. In a second step, it is necessary to observe in works representing the student spring how the anti-institutional ideology can be accompanied by a literary investment. In Le jeu de la musique (Stéfanie Clermont), Malabourg (Perrine Leblanc), Terre des cons (Patrick Nicol) and Qu'il est bon de se noyer (Cassie Bérard), we observe that this succession ideologically marked is contradicted by training literary from which it comes.
\end{abstract}

\title{
Notice biobibliographique
}

David Bélanger est chercheur postdoctoral à l'Université McGill et Chargé de cours à l'UQAM. Avec Jean-François Chassay et Michel Lacroix, il a codirigé deux volumes de la revue Voix et images consacrés au critique André Belleau. Avec Thomas Carrier-Lafleur, il a fait paraître un essai sur Maria Chapdelaine et son mythe, intitulé Il s'est écarté. Enquête sur la mort de François Paradis (Nota Bene, 2019). Il est directeur de rédaction d'XYZ. La revue de la nouvelle. 